\title{
The Analysis on the Singularity of a 2UPS-UPR Parallel Machine Tool
}

\author{
Liang Zhao ${ }^{1, a}$, Jianye $\mathrm{Guo}^{2}$ and Xiaohong $\mathrm{Li}^{1}$ \\ ${ }^{1}$ Liaoning Institute of Science and Technology, Benxi, China, 117004 \\ ${ }_{2}$ Shenyang Aerospace University, Shenyang, China, 110136
}

\begin{abstract}
This paper deals with the analysis on the singularity of a 2UPS-UPR parallel machine tool newly designed by Northeastern University. On the basis of establishing kinematics equations, the Jacobian matrix of the parallel machine tool and the shape of the parallel machine tool workspace are obtained, the degree of operability which is the determinant value of the product of Jacobian matrix and its inverse matrix is taken as the performance index of the singularity. With MATLAB software, the change situation about the degree of operability within the workspace is simulated. The analysis results of this paper have laid the theoretical foundation for the position control and processing track planning of the parallel machine tool.
\end{abstract}

\section{Introduction}

Parallel machine tools have drawn continuous interest in both industry and academia in the machine tool/robot sectors since the 1990s because of their potentially desirable fast dynamic performance, rigidity, and acceptable accuracy. It was clearly indicated that one of the future trends toward parallel machine tools' development is to make full use of its reconfigurability and multiple functionality[1]. Singularity is an inherent property of parallel machine tool that affects their performance greatly. When the parallel machine tool is in the singular position, its movement ability will be lost or out of control, which will directly cause the parallel machine tool can't work, so the singular position is very harmful and must be avoided vigorously[2-4]. Gosselin et al. studied singular configurations based on kinematic analysis, and classified these configurations into three types: boundary singularity, configuration singularity and compound singularity[5-6].

\section{Structure of the Parallel Machine Tool}

Here is the 2UPS-UPR parallel machine tool mechanism, illustrated in Fig.1. Two extensible legs 5, 6 are connected to the fixed support 1 by the universal joints 4 , 7 and the movable platform 8 by sphere joints respectively, the third extensible leg 10 is connected to the fixed support 1 by the universal joints 2 and the movable platform 8 by revolute pair respectively. A parallel-mechanism 3 consisting of two parallelogram structures is equipped between the movable platform 8 and the universal joint 2 . Owing to the restraint of parallel mechanism 3, the movable platform 8 has only three translational DOFs and one derived rotational DOF.
The angle between the horizontal plane and the normal of the movable platform 8 is designed to 45 degree. The milling head (electric spindle) 9 equipped on the movable platform 8, can rotate around the normal of the movable platform 8 , which leads to a result that the main shaft can work either vertically or horizontally.

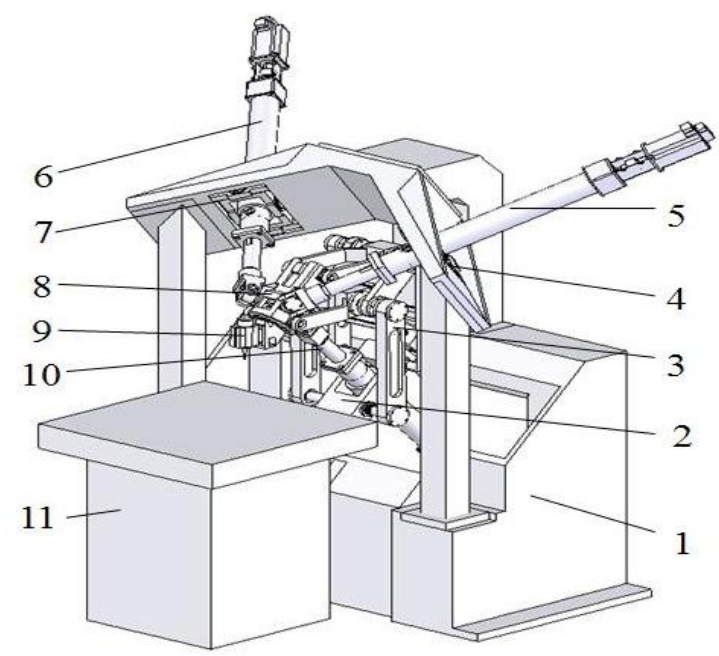

Figure 1. Structure of the 2UPS-UPR parallel machine tool. 1-fixed support, 2,4,7-universal joint, 3-parallelmechanism, 5,6,10-extensible leg, 8-movable platform, 9-milling head

\section{Jacobian Matrix of the Parallel Machine Tool}

Jacobian matrix is an important parameter for parallel machine tools. It refers to the generalized drive ratio of the movement velocity transmission from joint-space to operation-space, which is the basis on the research of

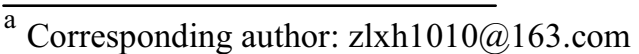


kinematics, statics, dynamics and error analysis etc. of parallel machine tools. Here, first, kinematic equations are set up by analyzing kinematics of parallel machine tools. Then, as the structure of the 2UPS-UPR parallel machine tool described in this paper is rather complex, it is comparatively difficult to obtain the position analytic positive solution, in order to avoid the complexity in obtaining Jacobian matrix from the position positive solution, so first to obtain the inverse matrix of Jacobian matrix of parallel machine tools from the position inverse solution, then to research kinematics and the movement performance on parallel machine tools as well.

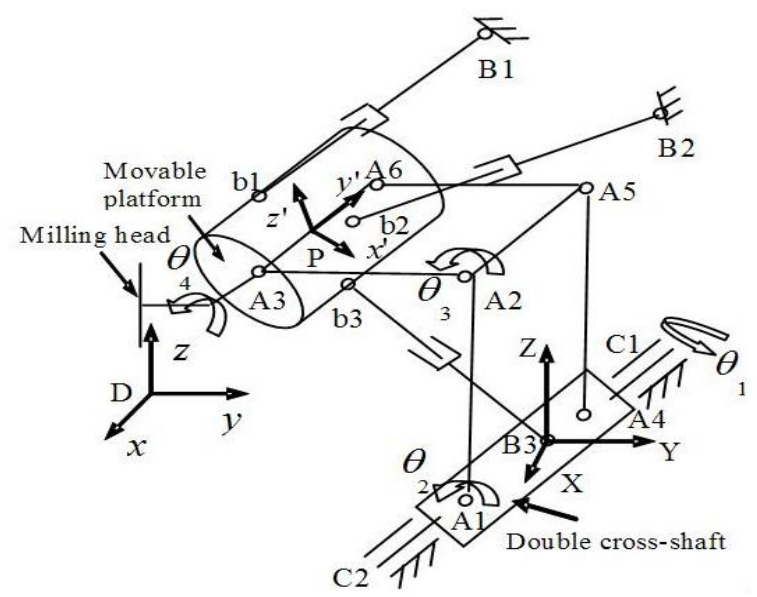

Figure 2. The 2UPS-UPR parallel machine tool schematic diagram.

Set up the fixed platform coordinate system $O-X Y Z$ and the movable platform coordinate system $P-x^{\prime} y^{\prime} z^{\prime}$ for the parallel machine tool, illustrated in Figure 2. Take $b_{i x}^{\prime} 、 b_{i y}^{\prime} 、 b_{i z}^{\prime}$ as the coordinate value of the joint point of movable platform in the movable platform coordinate system, and $B_{i X}$ 、

$B_{i Y} 、 B_{i Z}$ as the coordinate value of the joint point of fixed platform in the fixed platform coordinate system(where $i=1,2,3$ ). The coordinate value of tool tip $\mathrm{D}$ in the fixed platform coordinate system is $(x, y, z)$, and to make the tool tip on the extension line of the movable platform axis. The included angle between the rotary axis $C_{1} C_{2}$ of the double cross-shaft and the horizontal axis is $45^{\circ}$, so the inverse kinematics equations of Parallel Robot can be obtained as:

$\boldsymbol{L}_{\boldsymbol{i}}=\left[\begin{array}{c}b_{i x}^{\prime} \cos \theta_{1}+b_{i z}^{\prime} \sin \theta_{1}+x-B_{i X} \\ b_{i x}^{\prime} \sin \theta_{1} \sin 45^{\circ}-b_{i z}^{\prime} \cos \theta_{1} \sin 45^{\circ}+l_{d} \cos 45^{\circ}+y-B_{i Y} \\ -b_{i x}^{\prime} \sin \theta_{1} \cos 45^{\circ}+b_{i z}^{\prime} \cos \theta_{1} \cos 45^{\circ}+l_{d} \sin 45^{\circ}+z-B_{i Z}\end{array}\right]$

where, $i=1,2,3, \quad \theta_{1}=\operatorname{arctg} \frac{\sqrt{2} x}{z-y}, \quad l_{d}$ is the length of $P D$.

From (1) can be obtained:

$$
l_{i}=\sqrt{L_{i x}^{2}+L_{i y}^{2}+L_{i z}^{2}} \quad i=1,2,3
$$

Then, $\left(\begin{array}{lll}\dot{x} & \dot{y} & \dot{z}\end{array}\right)^{T}=J\left(\begin{array}{lll}\dot{l}_{1} & \dot{l}_{2} & \dot{l}_{3}\end{array}\right)^{T}, \quad$ that is, $\left(\begin{array}{lll}\dot{l}_{1} & \dot{l}_{2} & \dot{l}_{3}\end{array}\right)^{T}=J^{-1}\left(\begin{array}{lll}\dot{x} & \dot{y} & \dot{z}\end{array}\right)^{T}$

so, the inverse matrix of Jacobian matrix is:

$$
J^{-1}=\left[\begin{array}{lll}
\frac{\partial l_{1}}{\partial x} & \frac{\partial l_{1}}{\partial y} & \frac{\partial l_{1}}{\partial z} \\
\frac{\partial l_{2}}{\partial x} & \frac{\partial l_{2}}{\partial y} & \frac{\partial l_{2}}{\partial z} \\
\frac{\partial l_{3}}{\partial x} & \frac{\partial l_{3}}{\partial y} & \frac{\partial l_{3}}{\partial z}
\end{array}\right]
$$

The inverse matrix of Jacobian matrix can be obtained according to the inverse kinematics equations of parallel machine tools.

\section{The Workspace of the Parallel Machine Tool}

The workspace describes the working ability of the parallel machine tool from the geometric perspective, which is important index of evaluating the parallel machine tool performance and one of the important bases of mechanism design and movement planning[7]. At present, the analysis methods of the parallel machine tool workspace are two kinds: the analytic method and the numerical method.

Since it is difficult for the parallel machine tool to obtain the position analytic positive solution, the numerical method is depended on to search when determining its workspace. As much of the workspace internal points are searched out. Then the space shape composed of these points is the shape of the workspace. Specific method to search workspace is: First, a set of angle values of rotation pairs $\left(\theta_{1}, \theta_{2}, \theta_{3}\right)$ are obtained within the permissible range according to the restriction of parallel mechanism. Second, the tool tip point coordinates $(x, y, z)$ are obtained by the inverse

kinematics equations of parallel mechanism. Then, a set of length of extensible legs $\left(l_{1}, l_{2}, l_{3}\right)$ and angle of each joint corresponding to the tool tip point coordinates is obtained by the inverse kinematics equations of the parallel machine tool at this moment. Just determine this group of extensible legs' length and angle of each joint meets the limit constraints. If it meets the limit constraints, then we can say this point is in the workspace. Thus, the shape of the parallel machine tool workspace can be obtained as shown in Fig. 3 according to the actual design size of the parallel machine tool. It can be seen from the figure that the shape of the parallel machine tool workspace is irregular and the whole workspace is continuous and no empty hole. The actual limitative work area of the parallel machine tool is included in this space. 

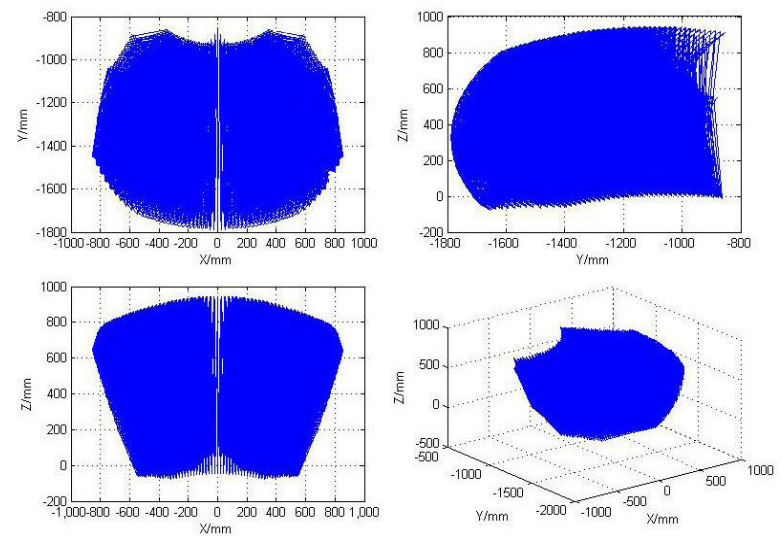

Figure 3. Diagram of workspace of the 2UPS-UPR parallel machine tool.

\section{Performance Index of the Singularity of the Parallel Machine Tool}

When the parallel machine tool is in some special positions that are called singular positions, the Jacobian matrix is singular matrix and the value of its determinant is zero, then the speed inverse solution of the parallel machine tool doesn't exist. The actual DOF of the parallel machine tool is no longer equal to the theoretical DOF in the critical condition when the parallel machine tool is at the singular position, and there may have two kinds of situations at this time: First, the parallel machine tool lost the proper DOF; second, the parallel machine tool obtained the additional DOF. The loss of parallel machine tool DOF means a certain loss of function; the acquisition of parallel machine tool additional DOF means that even if all the driver input is zero, the parallel machine tool can still move under the action of external force, thus resulting in the parallel machine tool to be out of control. Therefore the singular is very harmful and must be avoided for a parallel machine tool concerned[8].

The research methods of parallel machine tools singularity mainly have two kinds, namely algebraic method and geometric method. The algebraic method is that the Jacobian matrix of parallel machine tool is first established, and then the singular positions of parallel machine tool can be analyzed by making the determinant of Jacobian matrix is zero. The geometric method is that the singular positions of parallel machine tool can be judged by studying the rank of different liner vector subspace. The degree of operability is used to measure the singularity of parallel machine tool. The degree of operability refers to the determinant value of the product of Jacobian matrix and its inverse matrix, namely:

$$
W=W(J)=\sqrt{\operatorname{det}\left(J J^{T}\right)}
$$

\section{Numerical Simulation for the Singularity of the Parallel Machine Tool}

According to Eq.4, the change situation about the degree of operability within the workspace is simulated with MATLAB software, illustrated in Fig.4.
Fig.4(a) shows the change situation that the degree of operability changes within plane $\mathrm{YOZ}, \mathrm{X}=0$ of the workspace; Fig.4(b) shows the change situation that the degree of operability changes within plane YOZ, $\mathrm{X}=0$ of the workspace; Fig.4(c) shows the change situation that the degree of operability changes within plane $\mathrm{YOZ}, \mathrm{X}=0$ of the workspace.

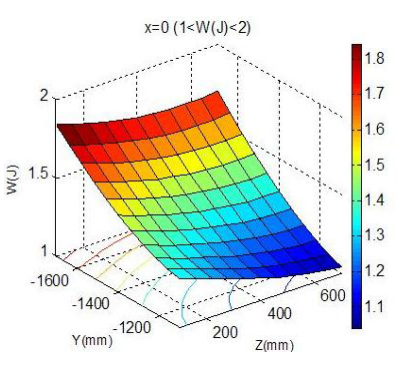

(a)

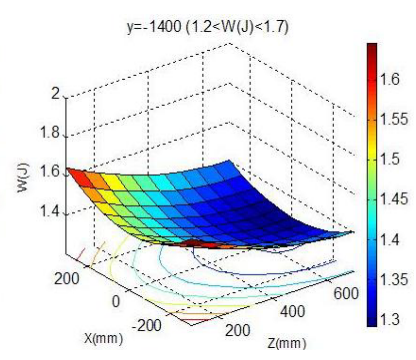

(b)

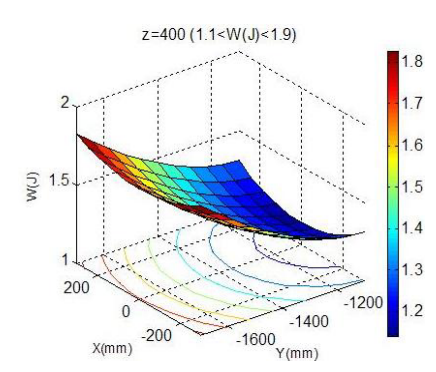

(c)

Figure 4. The change situation about the degree of operability.

It can be seen from Fig. 4 that $W \neq 0$, so the parallel machine tool does not have "dead center" within the workspace; and there is $W \neq \infty$, so the parallel machine tool does not have uncertain position within the workspace too, the change range of the degree of operability is: $1<W(J)<2$. Therefore, the singular position does not exist within the workspace of the parallel machine tool.

\section{Conclusion}

This paper takes the degree of operability as the performance index to study the distribution of a 2UPSUPR parallel mechanism tool singularity within the workspace based on establishing kinematics equations and obtaining Jacobian matrix. By analyzing the result of the simulation, we know that the change range of the degree of operability is: $1<W(J)<2$, and the singular position does not exist within the workspace of the parallel machine tool. The analysis results of this paper have laid the theoretical foundation for the position control and processing track planning of the parallel machine tool.

\section{Acknowledgements}


Supported by Program for Liaoning Excellent Talents in University, The doctoral scientific research fund project of Liaoning Institute of Science and Technology (1212B3)

\section{References}

1. M. Weck, D. Staimer, Ann. CIRP 51, 671-683 (2002)

2. J.P. Merlet, Parallel Robots. Kluwer Academic Publishers, Netherland (2000)

3. Z. Huang, L.F. Kong, Y.F. Fang, The Parallel Robot Mechanism and Control Theory. China Machine Press, China, (1997)
4. Y. L. Xiong, H. Ding, Robotics, China Machine Press, China, (1993)

5. Gosselin C, Angeles J, Transactions on Robotics and Automation, 6(3), 281-290 (1990)

6. Y.W. Zhang, G.H. Gui, Z.J. Sun et al, Transactions of the Chinese Society for Agricultural Machinery, 41, 903-906 (2010)

7. F. Pernkopf, M.L. Husty, Proceedings of MUSME 2002, Mexico City (2002)

8. J.Y. Guo, L. Zhao, J.S. Shi, Advanced Materials Research, 225-226, 903-906 (2011) 